\title{
THE PRODUCTION OF HIGH SPEED LIGHT IONS WITHOUT THE USE OF HIGH VOLTAGES
}

\author{
By Ernest O. Lawrence and M. Stanley Livingston \\ University of CALIFornia
}

(Received February 20, 1932)

\begin{abstract}
The study of the nucleus would be greatly facilitated by the development of sources of high speed ions, particularly protons and helium ions, having kinetic energies in excess of $1,000,000$ volt-electrons; for it appears that such swiftly moving particles are best suited to the task of nuclear excitation. The straightforward method of accelerating ions through the requisite differences of potential presents great experimental difficulties associated with the high electric fields necessarily involved. The present paper reports the development of a method that avoids these difficulties by means of the multiple acceleration of ions to high speeds without the use of high voltages. The method is as follows: Semi-circular hollow plates, not unlike duants of an electrometer, are mounted with their diametral edges adjacent, in a vacuum and in a uniform magnetic field that is normal to the plane of the plates. High frequency oscillations are applied to the plate electrodes producing an oscillating electric field over the diametral region between them. As a result during one half cycle the electric field accelerates ions, formed in the diametral region, into the interior of one of the electrodes, where they are bent around on circular paths by the magnetic field and eventually emerge again into the region between the electrodes. The magnetic field is adjusted so that the time required for traversal of a semi-circular path within the electrodes equals a half period of the oscillations. In consequence, when the ions return to the region between the electrodes, the electric field will have reversed direction, and the ions thus receive second increments of velocity on passing into the other electrode. Because the path radii within the electrodes are proportional to the velocities of the ions, the time required for a traversal of a semi-circular path is independent of their velocities. Hence if the ions take exactly one half cycle on their first semi-circles, they do likewise on all succeeding ones and therefore spiral around in resonance with the oscillating field until they reach the periphery of the apparatus. Their final kinetic energies are as many times greater than that corresponding to the voltage applied to the electrodes as the number of times they have crossed from one electrode to the other. This method is primarily designed for the acceleration of light ions and in the present experiments particular attention has been given to the production of high speed protons because of their presumably unique utility for experimental investigations of the atomic nucleus. Using a magnet with pole faces 11 inches in diameter, a current of $10^{-9}$ ampere of $1,220,000$ volt-protons has been produced in a tube to which the maximum applied voltage was only 4000 volts. There are two features of the developed experimental method which have contributed largely to its success. First there is the focussing action of the electric and magnetic fields which prevents serious loss of ions as they are accelerated. In consequence of this, the magnitudes of the high speed ion currents obtainable in this indirect manner are comparable with those conceivably obtainable by direct high voltage methods. Moreover, the focussing action results in the generation of very narrow beams of ions-less than $1 \mathrm{~mm}$ cross-sectional diameter-which are ideal for experimental studies of collision processes. Of hardly less importance is the second feature of the method which is the simple and highly effective means for the correction of the magnetic field along the paths of the ions. This makes it possible, indeed easy, to operate the tube effectively
\end{abstract}


with a very high amplification factor (i.e., ratio of final equivalent voltage of accelerated ions to applied voltage). In consequence, this method in its present stage of development constitutes a highly reliable and experimentally convenient source of high speed ions requiring relatively modest laboratory equipment. Moreover, the present experiments indicate that this indirect method of multiple acceleration now makes practicable the production in the laboratory of protons having kinetic energies in excess of 10,000,000 volt-electrons. With this in mind, a magnet having pole faces $114 \mathrm{~cm}$ in diameter is being installed in our laboratory.

\section{INTRODUCTION}

$\mathrm{T}$ HE classical experiments of Rutherford and his associates ${ }^{1}$ and Pose $^{2}$ on artificial disintegration, and of Bothe and Becker ${ }^{3}$ on excitation of nuclear radiation, substantiate the view that the nucleus is susceptible to the same general methods of investigation that have been so successful in revealing the extra-nuclear properties of the atom. Especially do the results of their work point to the great fruitfulness of studies of nuclear transitions excited artificially in the laboratory. The development of methods of nuclear excitation on an extensive scale is thus a problem of great interest; its solution is probably the key to a new world of phenomena, the world of the nucleus.

But it is as difficult as it is interesting, for the nucleus resists such experimental attacks with a formidable wall of high binding energies. Nuclear energy levels are widely separated and, in consequence, processes of nuclear excitation involve enormous amounts of energy-millions of volt-electrons.

It is therefore of interest to inquire as to the most promising modes of nuclear excitation. Two general methods present themselves; excitation by absorption of radiation (gamma radiation), and excitation by intimate nuclear collisions of high speed particles.

Of the first it may be said that recent experimental studies ${ }^{4,5}$ of the absorption of gamma radiation in matter show, for the heavier elements, variations with atomic number that indicate a quite appreciable nuclear effect. This suggests that nuclear excitation by absorption of radiation is perhaps a not infrequent process, and therefore that the development of an intense artificial source of gamma radiation of various wave-lengths would be of considerable value for nuclear studies. In our laboratory, as elsewhere, this being attempted.

But the collision method appears to be even more promising, in consequence of the researches of Rutherford and others cited above. Their pioneer investigations must always be regarded as really great experimental achievements, for they established definite and important information about nuclear processes of great rarity excited by exceedingly weak beams of bombarding particles-alpha-particles from radioactive sources. Moreover, and this is the point to be emphasized here, their work has shown strikingly the

${ }^{1}$ See Chapter 10 of Radiations from_Radioactive Substances by Rutherford, Chadwick and Ellis.

${ }^{2}$ H. Pose, Zeits. f. Physik 64, 1 (1930).

${ }^{3}$ W. Bothe and H. Becker, Zeits. f. Physik 66, 1289 (1930).

${ }^{4}$ G. Beck, Naturwiss. 18, 896 (1930).

${ }^{5}$ C. Y. Chao, Phys. Rev. 36, 1519 (1930). 
great fruitfulness of the kinetic collision method and the importance of the development of intense artificial sources of alpha-particles. Of course it cannot be inferred from their experiments that alpha-particles are the most effective nuclear projectiles: the question naturally arises whether lighter or heavier particles of given kinetic energy would be more effective in bringing about nuclear transitions.

A beginning has been made on the theoretical study of the nucleus and a partial answer to this question has been obtained. Gurney and Condon ${ }^{6}$ and Gamow $^{7}$ have independently applied the ideas of the wave mechanics to radioactivity with considerable success. Gamow ${ }^{8}$ has further considered along the same lines the penetration into the nucleus of swiftly moving charged particles (with excitation of nuclear transitions in mind) and has concluded that, for a given kinetic energy, the lighter the particle the greater is the probability that it will penetrate the nuclear potential wall. This result is not unconnected with the smaller momentum and consequent longer wavelength of the ligher particles; for it is well-known that transmission of matter waves through potential barriers becomes greater with increasing wavelengths.

If the probability of nuclear excitation by a charged particle were mainly dependent on its ability to penetrate the nuclear potential wall, electrons would be the most effective. However, there is considerable evidence that nuclear excitation by electrons is negligible. It suffices to mention here the current view that the average density of the extra-nuclear electrons is quite great in the region of the nucleus, i.e., that the nucleus is quite transparent to electrons; in other words, there are no available stable energy levels for them.

On the other hand, there is evidence that there are definite nuclear levels for protons as well as alpha-particles $;{ }^{9}$ indeed, there is some justification for the view that the general principles of the quantum mechanics are applicable in the nucleus to protons and alpha particles. It is not possible at the present time to estimate the relative excitation probabilities of the protons and alpha particles that succeed in penetrating the nucleus. However, it does seem likely that the greater penetrability of the proton* is an advantage outweighing any differences in their excitation characteristics. Protons thus appear to be most suited to the task of nuclear excitation.

Though at present the relative efficacy of protons and alpha-particles cannot be established with much certainty, it does seem safe to conclude at least that the most efficacious nuclear projectiles will prove to be swiftly moving ions, probably of low atomic number. In consequence it is important to develop methods of accelerating ions to speeds much greater than have heretofore been produced in the laboratory.

${ }^{6}$ Gurney and Condon, Phys. Rev. 33, 127 (1929).

${ }^{7}$ Gamow, Zeits. f. Physik 51, 204 (1928).

${ }^{8}$ Gamow, Zeits. f. Physik 52, 514 (1929).

${ }^{9}$ J. Chadwick, J. E. R. Constable, E. C. Pollard, Proc. Roy. Soc. A130, 463 (1930).

* According to Gamow's theory a one million volt-proton has as great a penetrating power as a sixteen million volt alpha-particle. 
The importance of this is generally recognized and several laboratories are developing techniques of the production and the application to vacuum tubes of high voltages for the generation of high speed electrons and ions. Highly significant progress in this direction has been made by Coolidge, ${ }^{10}$ Lauritsen, ${ }^{11}$ Tuve, Breit, Hafstad, Dahl, ${ }^{12}$ Brasch and Lange, ${ }^{13}$ Cockroft and Walton, ${ }^{14}$ Van de Graaff ${ }^{15}$ and others, who have developed several distinct techniques which have been applied to voltages of the order of magnitude of one million.

These methods involving the direct utilization of high voltages are subject to certain practical limitations. The experimental difficulties go up rapidly with increasing voltage; there are the difficulties of corona and insulation and also there is the problem of design of suitable high voltage vacuum tubes.

Because of these difficulties we have thought it desirable to develop methods for the acceleration of charged particles that do not require the use of high voltages. Our objective is two fold: first, to make the production of particles having kinetic energies of the order of magnitude of one million volt-electrons a matter that can be carried through with quite modest laboratory equipment and with an experimental convenience that, it is hoped, will lead to a widespread attack on this highly important domain of physical phenomena; and second, to make practicable the production of particles having kinetic energies in excess of those producible by direct high voltage methods-perhaps in the range of $10,000,000$ volt-electrons and above.

A method for the multiple acceleration of ions to high speeds, primarily designed for heavy ions, has recently been described in this journal. ${ }^{16}$ The present paper is a report of the development of a method for the multiple acceleration of light ions. ${ }^{17}$ Particular attention has been given to the acceleration of protons because of their apparent unique utility in nuclear studies. In the present work relatively large currents of 1,220,000 volt-protons have been generated and there is foreshadowed in the not distant future the production of $10,000,000$ volt-protons.

\section{The Experimental Method}

In the method for the multiple acceleration of ions to high speeds, recently described, ${ }^{16}$ the ions travel through a series of metal tubes in synchronism with an applied oscillating electric potential. It is so arranged that as an

${ }^{10}$ W. D. Collidge, Am. Inst. E. Eng. 47, 212 (1928).

${ }^{11}$ C. C. Lauritsen and R. D. Bennett, Phys. Rev. 32, 850 (1928).

12 M. A. Tuve, G. Breit, L. R. Hafstad and O. Dahl, Phys. Rev. 35, 66 (1930); M. A. Tuve, L. R. Hafstad, O. Dahl, Phys. Rev. 39, 384, (1932).

${ }^{13}$ A. Brasch and J. Lange, Zeits. f. Physik 70, 10 (1931).

${ }^{14}$ J. J. Cockroft and E. T. S. Walton, Proc. Roy. Soc. A129, 477 (1930).

${ }^{15}$ R. S. Van de Graaff, Schenectady Meeting American Physical Society, 1931.

${ }^{16}$ D. H. Sloan and E. O. Lawrence, Phys. Rev. 38, 2021 (1931).

${ }_{17}$ This method was first described before the September, 1930, meeting of the National Academy of Sciences (Lawrence and Edlefsen, Science 72, 376-377 (1930)). Later before the American Physical Society (Lawrence and Livingston, Phys. Rev. 37, 1707, (1931)) results of a preliminary study of the practicability of the method were given. Further work was reported in a Letter to the Editor of the Physical Review (Lawrence and Livingston, Phys. Rev. 38, 834 (1931). 
ion travels from the interior of one tube to the interior of the next there is always an accelerating field, and the final velocity of the ion on emergence from the system corresponds approximately to a voltage as many times greater than the applied voltage between adjacent tubes as there are tubes. The method is most conveniently used for the acceleration of heavy ions; for light ions travel faster and hence require longer systems of tubes for any given frequency of applied oscillations.

The present experimental method makes use of the same principle of repeated acceleration of the ions by a similar sort of resonance with an oscillating electric field, but has overcome the difficulty of the cumbersomely long accelerating system by causing, with the aid of a magnetic field, the ions to circulate back and forth from the interior of one electrode to the interior of another.

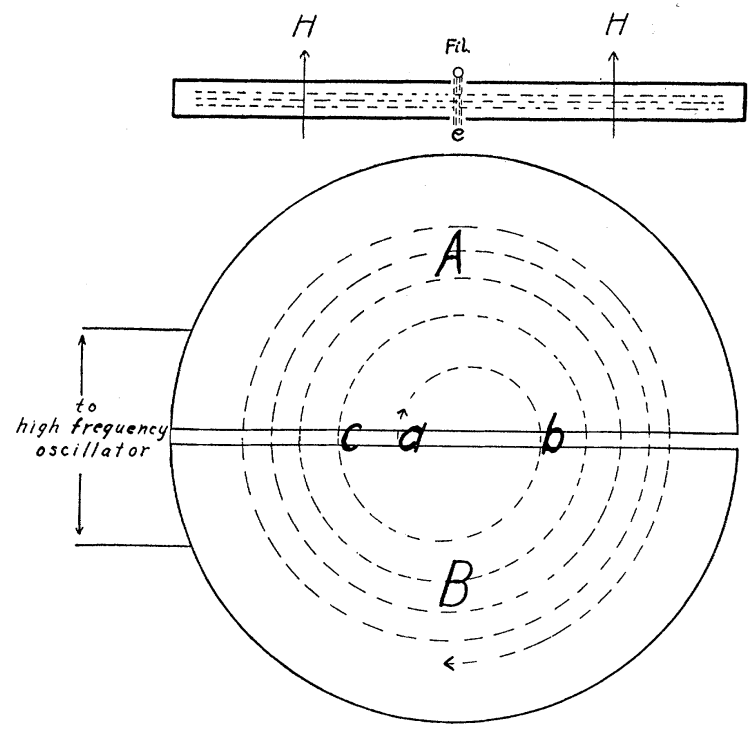

Fig. 1. Diagram of experimental method for multiple acceleration of ions.

This may be seen most readily by an outline of the experimental arrangement (Fig. 1). Two electrodes $A, B$ in the form of semi-circular hollow plates are mounted in a vacuum tube in coplanar fashion with their diametral edges adjacent. By placing the system between the poles of a magnet, a magnetic field is introduced that is normal to the plane of the plates. High frequency electric oscillations are applied to the plates so that there results an oscillating electric field in the diametral region between them.

With this arrangement it is evident that, if at one moment there is an ion in the region between the electrodes, and electrode $A$ is negative with respect to electrode $B$, then the ion will be accelerated to the interior of the former. Within the electrode the ion traverses a circular path because of the magnetic field, and ultimately emerges again between the electrodes; this is indicated in the diagram by the arc $a . . b$. If the time consumed by the ion in making the 
semi-circular path is equal to the half period of the electric oscillations, the electric field will have reversed and the ion will receive a second acceleration, passing in to the interior of electrode $B$ with a higher velocity. Again it travels on a semi-circular path $(b . . c)$, but this time the radius of curvature is greater because of the greater velocity. For all velocities (neglecting variation of mass with velocity) the radius of the path is proportional to the velocity, so that the time required for traversal of a semi-circular path is independent of the ion's velocity. Therefore, if the ion travels its first half circle in a half cycle of the oscillations, it will do likewise on all succeeding paths. Hence it will circulate around on ever widening semi-circles from the interior of one electrode to the interior of the other, gaining an increment of energy on each crossing of the diametral region that corresponds to the momentary potential difference between the electrodes. Thus, if, as was done in the present experiments, high frequency oscillations having peak values of 4000 volts are applied to the electrodes, and protons are caused to spiral around in this way 150 times, they will receive 300 increments of energy, acquiring thereby a speed corresponding to $1,200,000$ volts.

It is well to recapitulate these remarks in quantitative fashion. Along the circular paths within the electrodes the centrifugal force of an ion is balanced by the magnetic force on it, i.e., in customary notation,

$$
\frac{m v^{2}}{r}=\frac{H e v}{c} \text {. }
$$

It follows that the time for traversal of a semi-circular path is

$$
t=\frac{\pi r}{v}=\frac{\pi m c}{H e}
$$

which is independent of the radius $r$ of the path and the velocity $v$ of the ion. The particle of mass $m$ and charge $e$ thus may be caused to travel in phase with the oscillating electric field by suitable adjustment of the magnetic field $H$ : the relation between the wave-length $\lambda$ of the oscillations and the corresponding synchronizing magnetic field $H$ is in consequence

$$
\lambda=\frac{2 \pi m c^{2}}{H e} \text {. }
$$

Thus for protons and a magnetic field of 10,000 gauss the corresponding wavelength is 19.4 meters; for heavier particles the proper wave-length is proportionately longer.*

It is easily shown also that the energy $V$ in volt-electrons of the charged particles arriving at the periphery of the apparatus on a circle of radius $r$ is

* It should be mentioned that, for a given wave-length, the ions resonate with the oscillations when magnetic fields of $1 / 3,1 / 5$, etc., of that given by Eq. (3) are used. Such types of resonance were observed in the earlier experimental studies. In the present experiments, however, the high speed ions resulting from the primary type of resonance only were able to pass through the slit system to the collector, because of the high deflecting voltages used. 


$$
V=150 \frac{H^{2} r^{2}}{c^{2}} \frac{e}{m} .
$$

Thus, the theoretical maximum producible energy varies as the square of the radius and the square of the magnetic field.

\section{Experimental Arrangement}

The experimental arrangement is shown diagrammatically in some detail in Fig. 2. Fig. 3 is a photograph of the brass vacuum tube with cover removed showing the filament, the accelerating electrode, the deflecting plates and slit system, the probe in front of the first slit mounted on a ground joint and the Faraday collector behind the last slit. An external view of the apparatus is shown in Fig. 4. Here the tube is shown between the magnet pole faces, connected with the oscillator, the vacuum system and hydrogen generator. This gives a good general idea of the modest extent of the equipment involved for the generation of protons having energies somewhat in excess of 1,000,000 volt-electrons. The control panel and electrometer, being on the other side, are not shown in the picture. The description of the apparatus follows.

The accelerating system. Though there are obvious advantages in applying the high frequency potentials with respect to ground to both accelerating electrodes, in the present experiments it was found convenient to apply the high frequency voltage to only one of the electrodes, as indicated in Fig. 2. This electrode was a semi-circular hollow brass plate $24 \mathrm{~cm}$ in diameter and $1 \mathrm{~cm}$ thick. The sides of the hollow plate were of thin brass so that the interior of the plate had approximately these dimensions. It was mounted on a water-cooled copper re-entrant tube which in turn passed through a copper to glass seal. The electrode insulated in this way was mounted in an evacuated brass box having internal dimensions $2.6 \mathrm{~cm}$ by $28.6 \mathrm{~cm}$ by $28.6 \mathrm{~cm}$, there being thus a lateral clearance between the electrode and walls of the brass chamber of $8 \mathrm{~mm}$.

The brass box itself constituted the other electrode of the accelerating system. Across the mid-section of the brass chamber parallel to the diametral edge of the electrode $A$ was placed a brass dividing wall $S$ with slits of the same dimensions as the opening of the nearby electrode. This arrangement gave rise to the same type of oscillating electric fields as would have been produced had there been used two insulated semi-circular electrodes with their diametral edges adjacent and parallel.

The source of ions. An ideal source of ions is one that delivers to the diametral region between the electrodes large quantities of ions with low components of velocity normal to the plane of the accelerators. This requirement has most conveniently been met in the present experiments merely by having a filament placed above the diametral region from which a stream of electrons pass down along the magnetic lines of force, generating ions of gases in the tube. The ions so formed are pulled out sideways by the oscillating electric field. The electrons are not drawn out because of their very small radii of curvature in the magnetic field. Thus, the beam of electrons is col- 


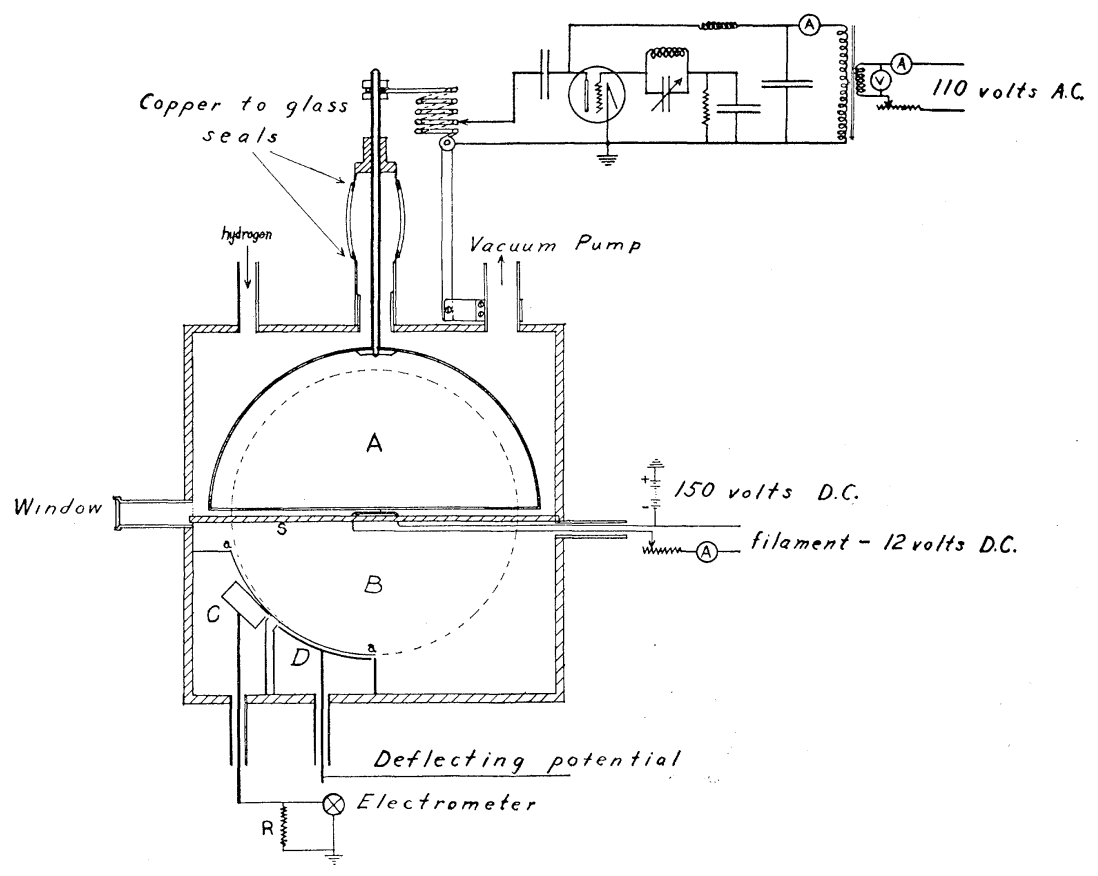

Fig. 2. Diagram of apparatus for the multiple acceleration of ions.

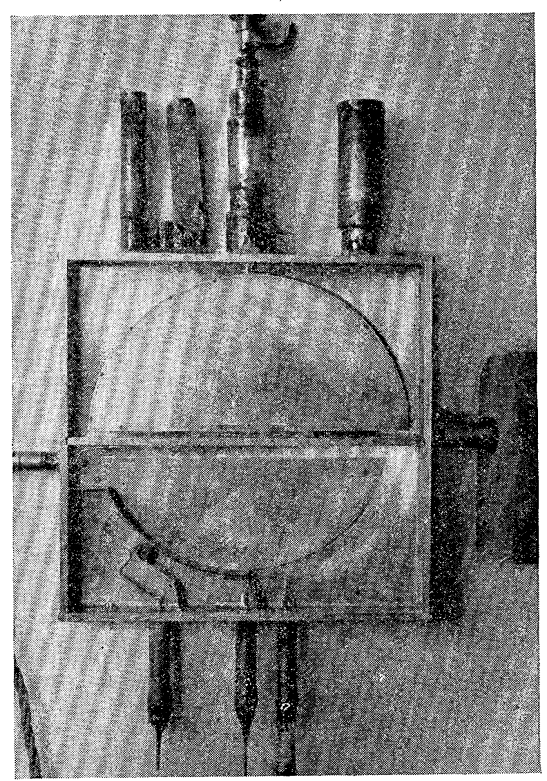

Fig. 3. Tube for the multiple acceleration of light ions-with cover removed. 
limated and the ions are formed with negligible initial velocities right in the region where they are wanted. The oscillating electric field immediately draws them out and takes them on their spiral paths to the periphery. This arrangement is diagrammatically shown in the upper part of Fig. 1.

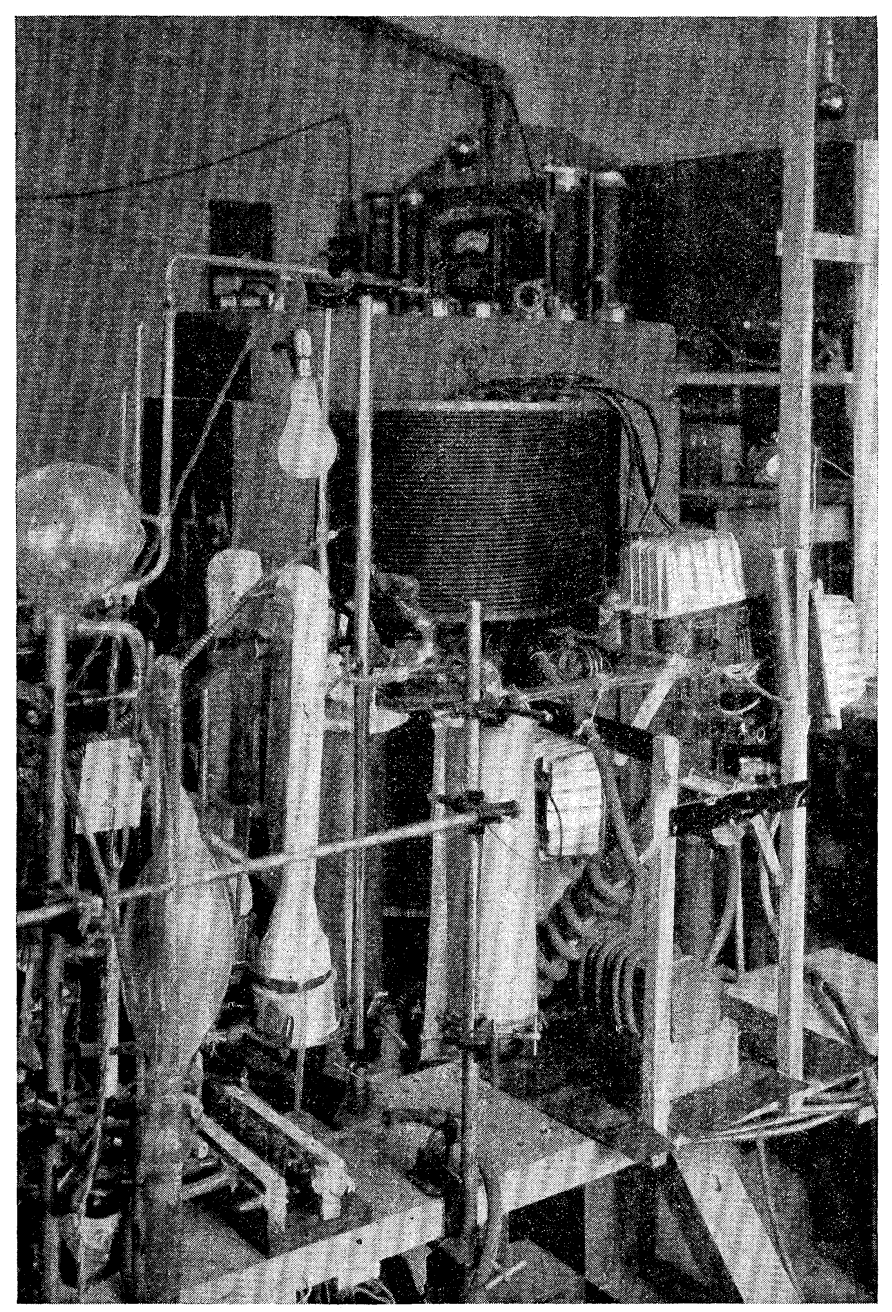

Fig. 4. External view of apparatus for generation of 1,220,000 volt protons.

The magnetic field. This experimental method requires a highly uniform magnetic field normal to the plane of the accelerating system. For example, if the ions are to circulate around 100 times, thereby gaining energy corresponding to 200 times the applied voltage, it is necessary that the magnetic field be uniform to a fraction of one percent. A general consideration of the matter leads one to the conclusion that, if possible, the magnetic field should be constant to about 0.1 percent from the center outward. Though this presumably 
difficult requirement has been met easily by an empirical method of field correction, the magnet used in the present experiments has pole faces machined as accurately as could be done conveniently. Its design was quite similar to that of Curtis. ${ }^{18}$ The pole faces were 11 inches in diameter and the gap separation was $1 \frac{1}{2}$ inches. Armco iron was used throughout the magnetic circuit. The magnetomotive force was provided by two coils of number 14 double cotton covered wire of 2,000 turns each. No water cooling was incorporated, for the magnet was not intended for high fields. In practice the magnet would give a field of 14,000 gauss for considerable periods without overheating. The pole faces were made parallel to about 0.2 percent and so it was to be expected that the magnetic field produced would be highly uniform. Exploration with a bismuth spiral confirmed this expectation, since it failed to show an appreciable variation of the magnetic field in the region between the poles, excepting within an inch of the periphery.

The collector system. In planning a suitable arrangement for collecting the high speed ions at the periphery of the apparatus, it was clearly desirable to devise something that would collect the high speed ions only and which would also measure their speeds. One might regard it as legitimate to suppose that the magnetic field itself and the distance of the collector from the center of the system would determine the speeds of the ions collected. This would be true provided there were no scattering and reflection of ions. To eliminate these extraneous effects a set of $1 \mathrm{~mm}$ slits was arranged on a circle $a . . a$, as shown in Fig. 2, of radius about 12 percent greater than the circle, indicated by the dotted line in the figure, having its center at the center of the tube and a radius of $11.5 \mathrm{~cm}$. The two circles were tangent at the first slit as shown. The ions on arrival at the first slit would be traveling presumably on circles approximately like the dotted line, and hence would not be able to pass through the second and third slits to the Faraday collector $C$. Electrostatic deflecting plates $D$, separated by $2 \mathrm{~mm}$, were placed between the first two slits, making possible the application of electrostatic fields to increase the radius of curvature of the paths of the high speed ions sufficiently to allow them to enter the collector. By applying suitable high potentials to the deflecting system in this way, only correspondingly high speed ions were registered.

The collector currents were measured by an electrometer shunted with a suitable high resistance leak.

The oscillator. The high frequency oscillations applied to the electrode were supplied by a 20 kilowatt Federal Telegraph water-cooled power tube in a "tuned plate tuned grid" circuit, for which the diagram of Fig. 2 is selfexplanatory.

\section{The Focussing Actions}

When one considers the circulation of the ions around many times as they are accelerated to high speeds in this way, one wonders whether in practice an appreciable fraction of those starting out can ever be made to

${ }^{18}$ L. F. Curtis, Jour. Op. Soc. Am. 13, 73 (1926). 
arrive at the periphery and to pass through a set of slits perhaps $1 \mathrm{~mm}$ wide and $1 \mathrm{~cm}$ long. The paths of the ions in the course of their acceleration would be several meters, and, because of the unavoidable spreading effects of space charge, thermal velocities and contact electromotive forces, as well as inhomogeneities of the applied fields, it would appear that the effective solid angle of the peripheral slit for the ions starting out would be exceedingly small.

Fortunately, however, this does not turn out to be the case. The electric and magnetic fields have been so arranged that they provide extremely strong focussing actions on the spiraling ions, which keep them circulating close to the median plane of the accelerating system.

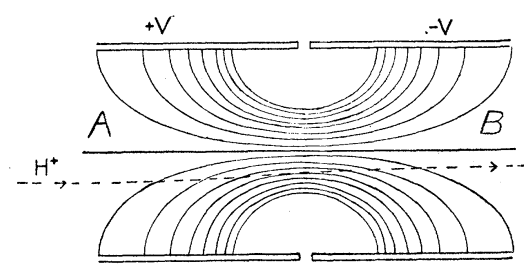

Fig. 5. Diagram indicating the focussing action of the electric field between the accelerating electrodes.

Fig. 5 shows the focussing action of the electric fields. There is depicted a cross-section of the diametral region between the accelerating electrodes with the nature of the field indicated by lines of force. There is shown also a dotted line which represents qualitatively the path of an ion as it passes from the interior of one electrode to the interior of the other. It is seen that, since it is off the median plane in electrode $A$, on crossing to $B$ it receives an inward displacement towards the median plane. This is because of the existence of the curvature of the field, which over certain regions has an appreciable component normal to the plane, as indicated. If the velocity of the ion is very

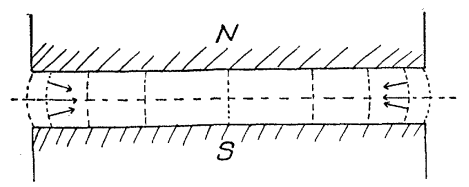

Fig. 6. Diagram indicating focussing action of magnetic field.

high in comparison to the increment of velocity gained in going from plate $A$ to plate $B$, its displacement towards the center will be relatively small and, to the first approximation, it may be described as due to the ion having been accelerated inward on the first half of its path across and accelerated outward by an equal amount during the remainder of its journey, the net result being a displacement of the ion towards the center without acquiring a net transverse component of velocity. In general, however, the outward acceleration during the second half will not quite compensate the inward acceleration of the first, resulting in a gain of an inward component of velocity as well as an inward displacement. In any event, as the ion spirals around it will migrate back and forth across the median plane and will not be lost to the walls of the tube. 
The magnetic field also has a focussing action. Fig. 6 shows diagrammatically the form of the field produced by the magnet. In the central region of the pole faces the magnetic field is quite uniform and normal to the plane of the faces; but out near the periphery the field has a curvature. Ions traveling on circles near the periphery experience thereby magnetic forces, indicated by the arrows. If the circular path is on the median plane then the magnetic force is towards the center in that plane. If the ion is traveling in a circle off the median plane, then there is a component of magnetic force that accelerates it towards the median plane, thereby giving effectively a focussing action.

We have experimentally examined these two focussing actions, using a probe in front of the first slit of the collector system that could be moved up and down across the beam by means of a ground joint (see Fig. 3). It was

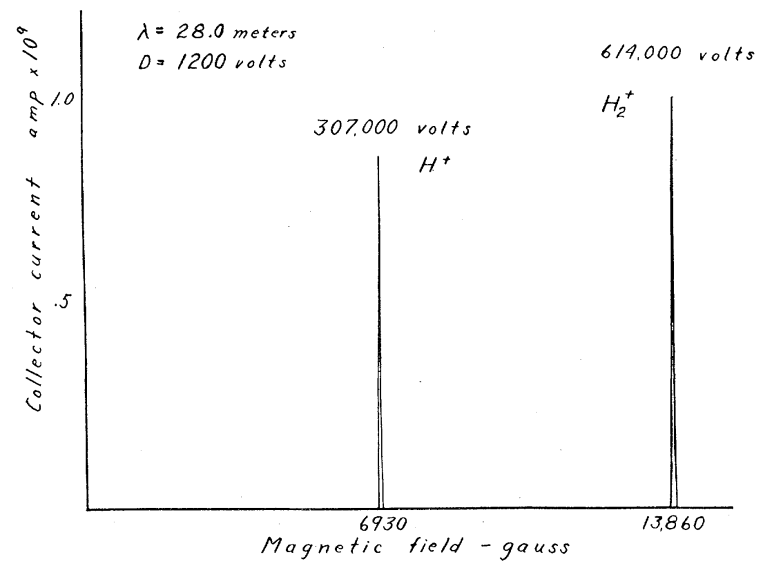

Fig. 7. Ion current to Faraday collector as a function of the magnetic field with oscillations of 28 meters wave-length applied to the accelerating electrodes.

found that the focussing actions were so powerful that the beam of high speed ions had a width of less than one millimeter. Such a narrow beam of ions of course is ideal for many experimental studies.

As a further test of the focussing action of the two fields, the median plane of the accelerating system was lowered $3 \mathrm{~mm}$ with respect to the plane of symmetry of the magnetic field. It was found that the high speed ion beam at the periphery traveled in a plane that was between the planes of symmetry of the two fields showing that both focussing actions were operative and at the periphery were of the same order of magnitude.

\section{EXPERIMENTAL RESUlts}

As a typical example there is shown in Fig. 7 a plot of the ion current to the Faraday collector as a function of the magnetic field for applied oscillations of wave-length 28 meters and with hydrogen in the tube. It is seen that there are only two narrow ranges of magnetic field strength over which ion currents are observed; both correspond exactly to expectations, the one at 
6930 gauss involving the resonance of protons, the other, hydrogen molecule ions.

For each wave-length used, the magnetic field giving the greatest current to the collector agreed precisely with the theoretically expected value. This is illustrated in Fig. 8 where the curves represent the theoretical hyperbolic relations between wave-length and magnetic field (Eq. 3) for protons and hydrogen molecule ions, and the circles represent the experimental observations. The magnetic fields were measured with a bismuth spiral and the oscillation wave-lengths were determined with a General Radio wavemeter. No effort was made to obtain considerable precision in these measurements, and in consequence their accuracy was hardly greater than 1 percent.

The variation with applied high frequency voltage of the widths of the resonance peaks agreed also with theoretical expectations. It was found that as the voltage was reduced the peaks became sharper, and indeed, with voltages such that the ions were required to spiral around fifty and more times to reach

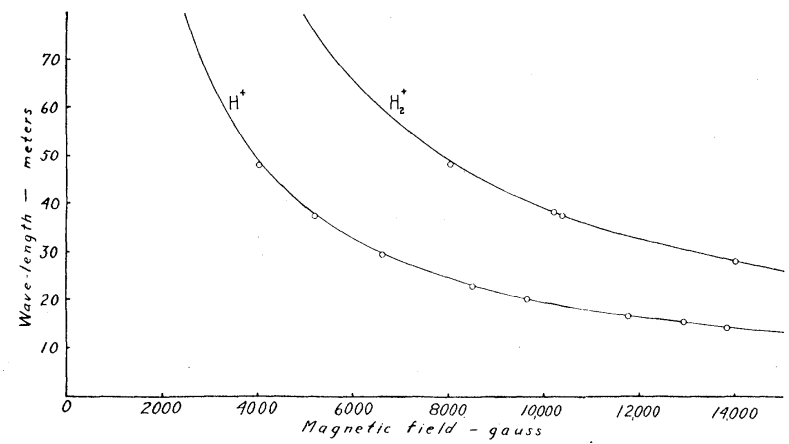

Fig. 8. Magnetic fields producing resonance of ions with oscillations of various wavelengths: the curves are the theoretical relations (Eq. (3)) for $\mathrm{H}^{+}$and $\mathrm{H}_{2}{ }^{+}$ions and the circles are the experimental observations.

the periphery, the ion currents diminished practically to zero when the magnetic field was changed a few tenths of one percent from the optimum value. This sharpness of resonance is understandable when it is remembered that the time required for an ion to execute one of its semi-circular paths is inversely proportional to the magnetic field. If, for example, the magnetic field were one percent greater or less than the resonance value, the ions would find themselves completely out of phase with the oscillations after having made fifty revolutions in the tube. In Fig. 7 the peaks exhibit an appreciable width, and indeed they extend over a one percent range of magnetic field. In most of the experiments, however, the ions circulated around many more times resulting in peaks of such restricted breadth as scarcely to be discernible in a diagram of this sort.

It is of course evident that the upper limit to the number of times the ions will circulate is determined by the degree of uniformity of the average value of the magnetic field along the spiral paths. Indeed, it would seem difficult to construct a magnet with pole faces giving fields of sufficient uniformity to 
allow more than 100 accelerations of the ions. But happily there is a very simple empirical way of correcting for the lack of uniformity of the field, that makes possible a surprisingly large voltage amplification. This is accomplished by insertion of thin sheets of iron between the tube and the magnet; either in the central region or out towards the periphery, as may be needed. If the magnetic field is, on the average, slightly less out towards the periphery so that the ions lag in phase more and more with respect to the oscillations as they spiral around, they may be brought back into step again by the insertion near the periphery of a strip of iron of suitable width, thickness and extension. If, on the other hand, the ions tend to get ahead in phase in this region, an effective correction can be made by inserting a suitable iron sheet in the central region.

It should be emphasized in this connection that the requirement is not that the magnetic field has to be uniform everywhere to the extent indicated above; small deviations from uniformity are allowable provided that the average value of the magnetic field over the paths of the ions is such that they traverse successive revolutions in equal intervals of time. Thus, small magnetic field adjustments can be accomplished by increasing or decreasing the field over small portions of successive circular paths of the ions. In the present experiments the most satisfactory adjustment was made by the insertion of a sheet of iron $0.025 \mathrm{~cm}$ thick having a shape much like an exclamation point extending radially with the thick end $8 \mathrm{~cm}$ wide in the central region and the narrow end $3 \mathrm{cms}$ wide at the periphery. Insertion of this correcting "shim" increased the amplification factor (that is, the ratio of the equivalent voltage of the ions arriving at the collector to the maximum high frequency voltage applied to the tube) from about 75 to about 300 . These figures are of necessity somewhat rough estimates, because no means were conveniently at hand to measure the high frequency voltages applied to the tube. Our estimates are based solely on sparking distances in air, and hence it is not unlikely that the voltage amplifications were even greater.

The greatest voltage amplification was obtained when generating the highest speed ions, 1,220,000 volt-protons. In all our work we have found the experimental method to be increasingly effective in this regard, as in others, as we go to higher voltages.

For example, the optimum pressure of hydrogen in the tube has been found to increase from less than $10^{-4} \mathrm{~mm}$ of $\mathrm{Hg}$ when generating 200,000 volt-protons to more than $10^{-3} \mathrm{~mm}$ when producing $1,000,000$ volt-protons. By the optimum pressure is meant the pressure that gives the largest current to the collector for a given electron emission from the filament. The reason for this is, of course, connected with the fact that the effective mean free path of the spiralling particles increases with voltage.

Examples of the observed variation with voltage on the deflecting plates of the ion currents to the collector are shown in Fig. 9. Each curve is for a particular resonance condition; curve $A$, for example, was obtained when protons resonated with 37.5 meter oscillations in a magnetic field of 5180 gauss, thereby theoretically resulting in the arrival of 172,000 volt-protons 
at the first slit of the collector system. The wave-lengths used and the theoretically expected equivalent voltages of the ions generated in each instance is indicated in the figure. It is seen that, the higher the equivalent voltage of the ions, the higher was the required deflecting voltage to obtain the maximum ion currents to the collector. Indeed, within the experimental error, the optimum deflecting voltage was proportional to the theoretical kinetic energies of the ions (calculated from Eq. (4)) and was quite independent of the magnitude of the high frequency voltage applied to the accelerating electrode. These observations constitute incontrovertible evidence that the ions arriving at the collector actually had the high speeds theoretically expected. The observed absolute magnitudes of the deflecting voltages also agreed with theoretical calculations within the experimental uncertainty of the paths of the ions before entering the deflecting system. Because of the considerable width of the ion source (the filament was $2.5 \mathrm{~cm} \mathrm{long}$ ) the effective center of

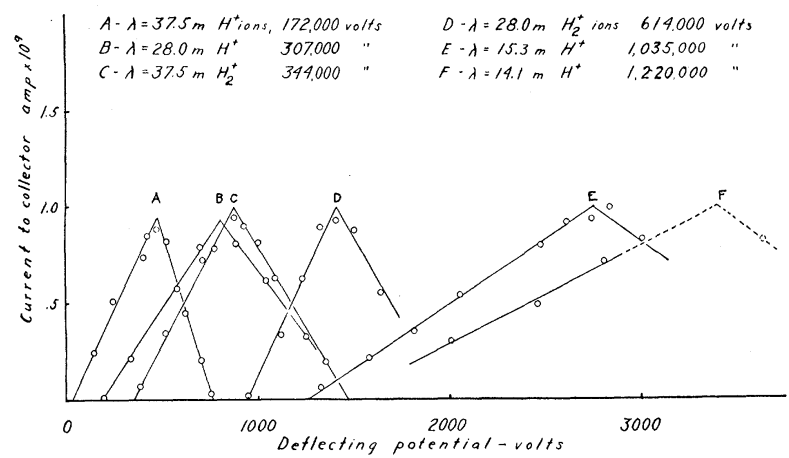

Fig. 9. Ion currents to the Faraday collector as a function of the voltage applied to the deflecting plates. The optimum deflecting voltages are seen to be proportional to the theoretically calculated kinetic energies of the ions (indicated in the figure in volts), thus proving that the ions arriving at the collector actually have the theoretically expected high speeds.

the circular paths of the ions at the periphery was quite broad. This fact together with the slit widths accounted for the absolute range of deflecting voltages over which ion currents reached the collector.

\section{Discussion}

The present experiments have accomplished one of the objectives set forth in the introduction, namely, the development of a convenient method for the production of protons having kinetic energies of the order of magnitude of $1,000,000$ volt-electrons. It is well to emphasize two particular features that have contributed more than anything else to the effectiveness of the method: the focussing actions of the electric and magnetic fields, and the simple means of empirically correcting the magnetic field by the introduction of suitable iron strips. The former has solved the practical problem of generation of intense high speed ion beams of restricted cross-section so much desired in studies of collision processes. The latter has eliminated the problem of uniformity of magnetic field, making possible voltage amplifications of more than 300 . This in turn has practically eliminated any difficulties associated 
with generation and application to the accelerating electrodes of required high frequency voltages. In consequence, we have here a source of high speed light ions that is readily constructed and assembled in a relatively small laboratory space out of quite modest laboratory equipment. The beam of ions so produced has valuable characteristics of convenience and flexibility for many experimental investigations; there are obvious advantages of a steady beam of high speed ions of but one millimeter diameter generated in an apparatus on an ordinary laboratory table. Moreover, the apparatus evolved in the present work is in no respects capricious, but functions always in a satisfactorily predictable fashion. This is illustrated by the fact that the accelerating tube can be taken apart and reassembled, and then within a few hours after re-evacuation steady beams of $1,200,000$ protons can always be obtained.

But it is perhaps of even more interest to inquire as to the practical limitations of the method; to see what extensions and developments are foreshadowed by the present experiments.

Of primary importance is the probable experimental limitation on the producible proton energies. The practical limit is set by the size of the electromagnet available; for the final equivalent voltage of the ions at the periphery is proportional to the square of the magnetic field strength and to the square of the radius of the path. For protons, it is not feasible to use magnetic fields much greater than employed in the present work (about 14,000 gauss) because of the difficulties of application of suitably higher frequency oscillations-that is to say, it is not desirable to go much below 14 meters wavelength. However, it is entirely practicable to use a much larger magnet than that employed in the present experiments. At the present time a magnet having pole faces $114 \mathrm{~cm}$ in diameter is being installed in our laboratory. As will be seen from Eq. (4), a magnetic field of 14,000 gauss over such a large region makes possible the production of $25,000,000$ volt-protons.

Of course, it may be argued that there are other difficulties which preclude ever reaching such a range of energies. For example, there is the question of whether it is possible to obtain such a great amplification factor that the high frequency voltages necessarily applied to the accelerating electrodes are low enough to be realizable in practice. In the present experiments an amplification of 300 was obtained with no great effort, and it would seem that with more careful correction of the field this amplification could be considerably increased at higher voltages. In the higher range of speeds the variation of mass with velocity begins to be appreciable, but presents no difficulty as it can be allowed for by suitable alteration of the magnetic field in the same empirical manner as is done to correct its otherwise lack of uniformity.

Assuming then a voltage amplification of 500 , the production of 25,000 ,000 volt-protons would require 50,000 volts at a wave-length of 14 meters applied across the accelerators; thus, 25,000 volts on each accelerator with respect to ground. It does appear entirely feasible to do this, although to be sure a considerable amount of power would have to be supplied because of the capacity of the system. 
Of similar interest is the matter of maximum obtainable beam intensities. In the present experiments no efforts have been made to obtain high intensities and the collector currents have usually been of the order of magnitude of $10^{-9} \mathrm{amp}$. Using the present method of generation of the ions, there are two factors that can be drawn upon to increase the yield of high speed ions - the electron emission and the pressure of hydrogen in the tube. The electron emission can easily be increased from 10 to 100 times over that used in the present experiments. The effective free paths of the protons increase with voltage so that, as was found to be the case, the maximum usable pressure of hydrogen is governed by the setting in of a high frequency discharge in the tubes due to the voltage on the accelerators. This appears to occur at a pressure greater than $10^{-3} \mathrm{~mm}$ of $\mathrm{Hg}$; the reason the critical pressure is so high is probably to be associated with the quenching action of the magnetic field. These considerations make it seem reasonable to expect that, using the present ion source, high speed ion currents of as much as 0.1 microampere can readily be obtained.

At all events, it seems that the focussing of the spiralling ions is so effective that a quite considerable portion of those starting out arrive at the collector and that the beam intensity is determined largely by the source. This method of multiple acceleration is capable of yields of the same order of magnitude as would conceivably result from the direct application of high voltages.

For a given experimental arrangement the energy of the ions arriving at the collector varies inversely as their masses and directly as their charges. Thus, the large magnet mentioned above makes possible the production of $12,500,000$ volt hydrogen molecule ions and doubly charged helium ions (alpha-particles) as well as 25,000,000 volt-protons. Moreover, generating the theoretically maximum value of ion energies becomes much easier with increasing atomic weight because the wave-length of the applied high frequency oscillations increases in a like ratio. For example, using a magnetic field of 14,000 gauss over a region $114 \mathrm{~cm}$ in diameter, 2,800,000 volt nitrogen ions could be generated by applying 123 meter oscillations. Broadly speaking, then, the apparatus is well adapted to the production of ions of all the elements up to atomic weight 25 having kinetic energies in excess of $1,000,000$ volt-electrons.

We wish to express our gratitude and thanks to the Committee-on-Grantsin-Aid of the National Research Council, the Federal Telegraph Company through the courtesy of Dr. Leonard F. Fuller, Vice-President, the Research Corporation, and the Chemical Foundation for their generous assistance which has made these experiments possible. 


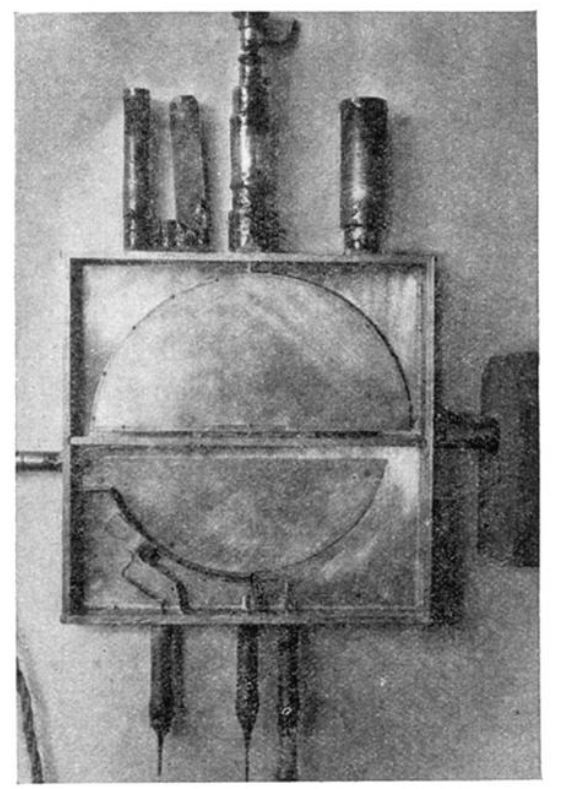

Fig. 3. Tube for the multiple acceleration of light ions-with cover removed. 


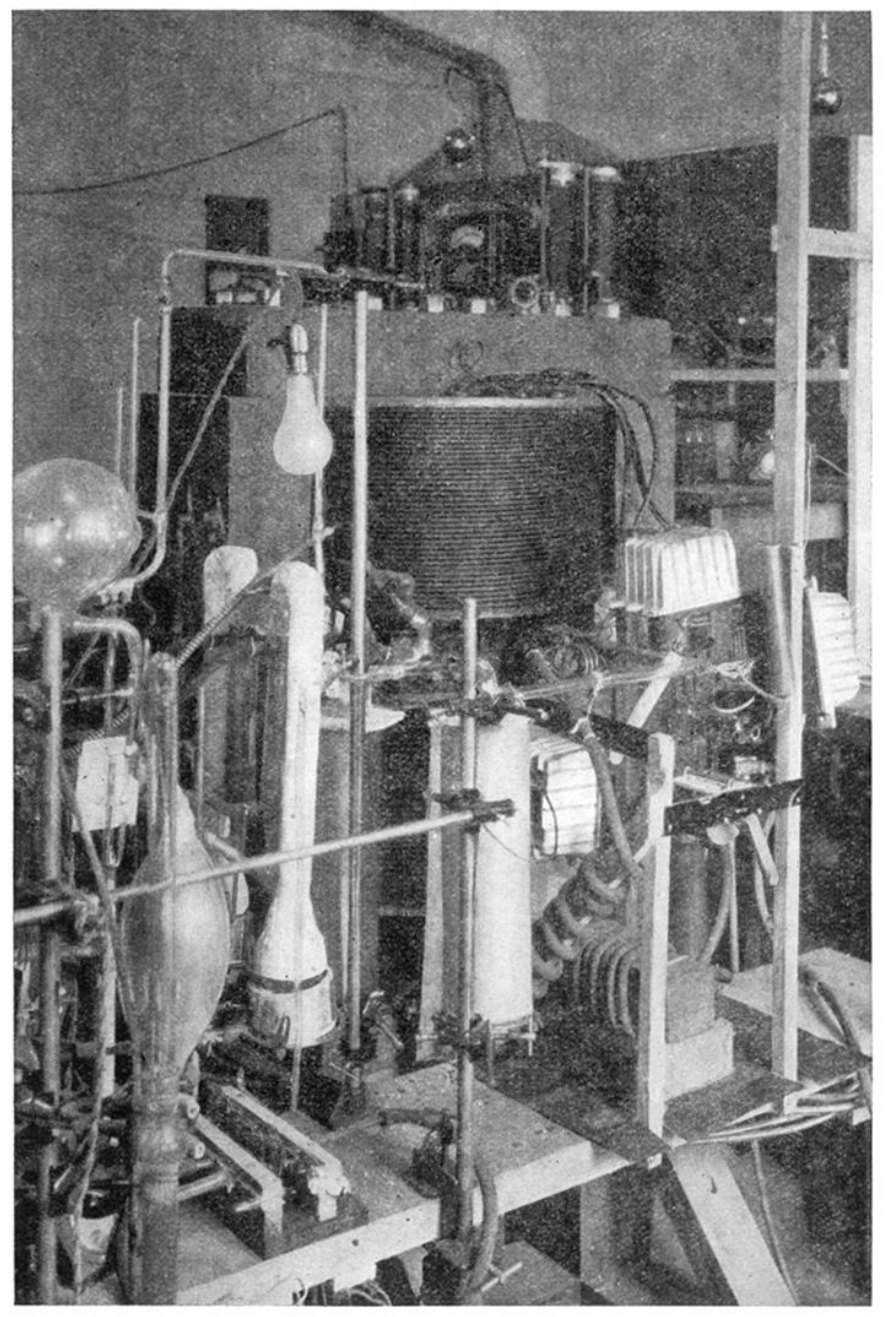

Fig. 4. External view of apparatus for generation of $1,220,000$ volt protons. 